PART I

\title{
FORMATION AND EVOLUTION \\ OF \\ DISC GALAXIES
}


FORMATION OF SPIRAL GAIAXIES

Richard B. Larson

Yale Untversity Observatory

\section{INTRODUCTION}

Conventionally, two major components of spiral galaxies are distinguished: (1) a "spheroidal component", which includes the central bulge and the surrounding halo, and (2) a "disc component", which is basically flat and circular but is often warped at the edges. Other structures, such as bars or rings, may also be present, but they are probably of less fundamental significance for the processes of galaxy formation, and therefore they will not be considered here. The distinction between bulge and disc components corresponds approximately to the classical distinction between Population II, as exemplifled by the globular clusters which have a nearly spherical distribution, and Population $I$, which is confined to the galactic plane and is relatively young and metal-rich. However, unlike the "classical" Population II objects which are old and metal-poor, the inner part of the bulge of our galaxy and of other galaxies is in fact metal-rich, and may even contain some young stars.

It is widely accepted, at least as a working hypothesis, that the spheroidal components of spiral galaxies are simflar in all respects to elliptical galaxies of comparable slze and mass; present data do not contradict this hypothesis. A spiral galaxy may then be described as an elliptical galaxy plus a disc component, whose properties and origin are not necessarily closely related to those of the elliptical component. We can therefore conveniently discuss separately the formation of the spheroidal and disc components of spiral galaxies, keeping open for the time being the question of the relation between them.

\section{GRAVITATIONAL COLLAPSE OF PROTOGALAXIES}

Since it is widely belfeved that galaxies form by some sort of gravitational collapse or accumulation process, it is useful to consider first some general properties of gravitational collapse. Numerical 
calculations always result in a very nonuniform collapse characterized by the runaway growth of any density fluctuations, whether they are present initially or whether they propagate inward from the boundary. Thus a collapsing cloud may be expected to develop a very lumpy structure, with dense condensations embedded in a more diffuse medium. This is indicated in some recent calculations of 3-dimensional isothermal collapse made with a finite-particle scheme (Larson 1978a,b); the number of condensations formed is found to be approximately equal to the number of Jeans masses in the initial cloud, as might be expected. The condensations rapidly collapse and develop dense cores which then proceed to accrete the gas around them; in general, because of the angular momentum of the accreted material, this leads to the formation of accretion discs around the dense cores.

In a collapsing protogalaxy, the temperature may be near $10^{4} \mathrm{~K}$ for a wide range of conditions, implying Jeans masses in the range i $10^{7}-10^{9} \mathrm{M}_{\odot}$. Condensations with masses of this order may then be expected to form and to begin collapsing on their own. After a sufficient increase in density, rapid cooling will eventually occur and reduce the temperature in the collapsing condensations to far below $10^{4} \mathrm{~K}$, great $1 \mathrm{y}$ reducing the Jeans mass and allowing the formation of large numbers of stars. Thus the first structures to appear in a collapsing protogalaxy may be subsystems of gas and stars having the size of small galaxies. If these subsystems have a suffictent density contrast, they may develop into well-formed separate galaxies. However, tidal interactions between them will eventually become important and cause them to merge into a single larger system, as emphasized in different contexts by Toomre (1977) and Ostriker (1977). Dynamical friction will cause the densest parts of the subsystems to spiral together to form a dense core, while the less dense parts are stripped off to form a diffuse envelope, producing ultimately a smooth, centrally condensed, more or less spheroidal system of stars. If the gas which has not yet turned into stars is retained in the system, it w11l eventually dissipate its random motions and settle into a disc.

Thus one can understand in a general way how galaxies with both spheroidal and disc components can form: the spheroidal component forms from stars made in dense subsystems before interactions and gaseous dissipation processes have become very important, while the disc forms from whatever gas remains at later times to experience further dissipation. The processes involved in the formation of spheroidal and disc systems will be considered in more detall below. It can be noted here, however, that the processes of tidal disruption and coalescence mentioned above are predicted to occur eventually for any gravitationally bound system of subunits, such as a cluster of galaxies; we still see separate galaxies in bound clusters only because the timescale for coalescence is 1onger than the present age of the universe, but eventually all of the galaxies in a system like the Local Group (if it is bound) will merge into a single large galaxy. Many peculiar and interacting galaxies may represent various stages of 
such tidal interaction and coalescence processes (Toomre 1977).

\section{FORMATION OF SPHEROIDAL SYSTEMS}

All of the models that have been proposed for the formation of elliptical galaxies share the common requirement that most of the stars must form early, 1.e. before there has been too much interaction between different parts of the system (Larson 1976b, Gott 1977). Rapid early star formation is plausible only in dense condensations or subsystems, so we are led again to the picture of a very nonuniform protogalaxy. An extreme case is represented by the suggestion of Toomre (1977) that elliptical galaxies may often origlnate from mergers between a small number of disc galaxies in which most of the gas has already been turned into stars. However, since purely stellar models do not directly explain the highly condensed cores or the metallicity gradients frequently observed in elliptical galaxies, it is probably necessary to assume that some gas remains after the initial coalescence of subsystems and forms metal-enriched stars with a more centrally condensed spatial distribution, as in the models of Larson (1975).

Eventually, stellar dynamical effects will smooth out most of the initial lumpiness in the distribution of stars; the larger and more massive subsystems will suffer tidal disruption and coalescence, while the smallest ones will have short relaxation times and thus will soon evaporate. After $10^{10}$ years only dense clusters of intermediate mass, such as the presently observed globular clusters, can survive (Rees 1977), and even these will eventually disappear. Thus the globular clusters may represent the last surviving remnants of the initially very nonuniform structure of the galactic halo.

It would be of interest to know how many subsystems were initially present in our own galaxy, how much mass they contained, and how star formation and metal enrichment processes occurred in them. The fact that the globular clusters in the outer halo show a great dispersion in metal abundances which is not correlated with position suggests that they formed in many separate "fragments" that evolved independently and achieved different degrees of metal enrichment (Searle 1977). On this picture the spread in metallicities of the globular clusters can be explained explained as a result of statistical fluctuations if star formation occurred in bursts and if each fragment experienced only a few such bursts (Searle 1977). These bursts of star formation and metal enrichment might even be identiflable with the formation of individual globular clusters. Only a small fraction of the total galactic mass may have been involved in the formation of the inftial fragments, since only a small fraction of the present visible mass of our galaxy is in the halo. 


\section{FORMATION OF DISCS}

Disc systems can form only from material that remains in gaseous form long enough for nearly all of its random motion to be dissipated. This might include both gas swept out of the original subsystems by collisions between them, and diffuse outlying gas that falls in later; a further source of gas may be mass loss from halo stars. Most traces of the initial nonuniform distribution of the gas are probably destroyed by the strong dissipative processes associated with the formation of the disc, so It may not be possible to infer from the present properties of the galactic disc much about the origin or spatial distribution of the gas from which it formed. However, the timescale for disc formation, i.e. the time dependence for the infall of gas into the galactic plane, is of great importance for galactic evolution, and may bear directly on the chemical properties of disc stars. In discussing the timescale of disc formation, it will be useful to consider first two possible limitIng models for the formation of discs.

One limiting case is the rapid collapse of a unfform protogalactic gas cloud, for example a uniform spheroid of gas that collapses in free fall to a disc. This is essentially the model proposed by Eggen, Lynden-Be11, and Sandage (1962, hereafter ELS). In this case all of the gas arrives in the disc at almost the same time, and the disc thereafter evolves as a closed system without further addition of mass. If star formation and metal production occur during the collapse, the progressive enrichment of the collapsing cloud leads to a spatlal gradient in the average metallicity of the stars formed, such that the metallicity decreases with increasing height above the galactic plane. Moreover, since the formation of the disc must be accompanied by the dissipation of random motions in the gas, the velocity dispersion of newly formed stars decreases with time, leading to an inverse correlation between velocity dispersion and metallicity that is in qualitative agreement with that observed for solar neighborhood stars. The observed correlation between velocity dispersion and metallicity was taken by ELS as evidence that our galaxy did in fact collapse during its early history. It may be noted, however, that this interpretation of the observations can be applied only to stars that actually formed during the initial rapid collapse, and not to the majority of disc stars which formed later.

ELS supposed that the initial protocloud was basically uniform and quiescent, each part having only a circular motion of rotation, and they argued that the present large orbital eccentricities of some stars can be explained only if their initially circular orbits were altered by a rapid change in the galactic potential field caused by a rapid collapse lasting no longer than $\sim 2 \times 10^{8} \mathrm{yr}$. If, however, stars were initially formed with large non-circular velocities, as seems much more likely in an Irregular protogalaxy, this argument no longer applies and the collapse time could in principle be much longer. Slower collapse and disc formation could result if dissipation processes are slow, as in some of the models of Larson (1976a), or if gas continues to fall in 
from great distances over a long period of time, as in the "cosmological infa11" picture of galaxy formation (Oort 1970, Larson 1972, Gott 1977).

Thus, as an opposite Iimiting case, we can consider the possibility that the disc forms by gradual infall of primordial gas, the infall continuing at a steady rate throughout the 11fetime of the galaxy. In this case a vertical metallicity gradient is again established because of the progressive enrichment of the infalling gas as it flows into the galactic plane. Likewise a correlation between velocity dispersion and metallicity is predicted because stars formed farther away from the galactic plane have a higher velocity dispersion. It is important to note that in the infall picture these correlations result largely or entirely from spatial gradients in a system with a steady gas flow, rather than from time varfations in the properties of a homogeneous collapsing cloud, as before. These spatial gradients can even be independent of time, as is 1llustrated by some of the results obtained for spherical collapse models by Larson (1974). Thus it is important to determine to what extent the observed trends reflect time variations and to what extent they result from spatial gradients present at a given time.

Infall or gas flow models possess some advantages over simple closed models of galactic chemical evolution, since they characteristically predict a slow varfation of metallicity with time; this might explain the fact that the solar neighborhood has fewer metal-poor stars and a narrower metallicity distribution than is predicted by the simplest closed models (Audouze and Tinsley 1976). Also, an inward flow of gas in the galactlc disc could contribute to explaining the radial abundance gradient in the disc (Tinsley, this colloquium).

A variant of this type of model is the halo infall model proposed by Ostriker and Thuan (1975), in which part of the disc gas is supplied by mass loss from halo stars. In this case, most of the infall occurs at early times and is strongly enriched by the nucleosynthesis products of massive halo stars; later, both the infall rate and the metallicity of the infalling gas decline as mass loss from less massive stars becomes the dominant contributor. This type of model has the additional advantage that the early infall of already enriched gas enhances the rapid initial rise of metallicity in the disc, and thus helps to account for the observed paucity of metal-poor stars.

The effects discussed above are all present to varying degrees in the hydrodynamic collapse models of Larson (1976a), in which star formation and dissipation take place concurrently in a contracting protogalaxy, forming first a spheroidal component and later a disc. In these models the disc is formed by infall at a decreasing rate of both primordial gas and gas lost from halo stars; halo infall 1s most important in the inner part of the disc, while the outer disc forms mostly from primordial gas which falls in from greater distances and settles Into a disc over a longer period of time. With appropriate 
choices of the assumptions and model parameters, particularly those governing the star formation rate, these models produce a two-component structure like that of many spiral galaxies, with most of the mass in the disc component. In addition, they predict a radial metallicity gradient in at least the inner part of the halo, and a steep metallicity gradient perpendicular to the plane of the disc, both in qualitative agreement with observations for our galaxy.

Improved calculations of the chemical evolution of the disc components of some of these models have recently been made by Tinsley and Larson (1978). At all radii, the metallicity $z_{g}$ of the gas rises rapidly at first because of the infall of gas pieviously enriched by massive halo stars, as in the Ostriker-Thuan (1975) models. This initial rapid increase in $z_{g}$ is most pronounced at small radii, where there is a brief "super-metal-rich" peak in metallicity before halo star formation subsides and $\mathrm{z}_{\mathrm{g}}$ drops again. Later, as disc star formation becomes important, $\mathrm{Z}_{\mathrm{g}}$ rises more slowly and eventually approaches a nearly constant value at most radil, owing to the continuing infall of metal-poor gas. The resulting radial gradients in metallicity for both gas and stars are fairly shallow in the inner disc where the gas fraction is small, but become steeper in the outer part of the disc where the gas fraction increases considerably due to slower star formation. The predicted steepening of the stellar metallicity gradient at large radii is similar to the observational results of Janes (this colloquium) for disc stars in our galaxy. The predicted metallicity gradient of the gas is smaller than that derived by Peimbert (this colloquium), but is very sensitive to the amount of continuing gas infal1; if infall at late times is suppressed, for example by a galactic wind, a steeper gradient of $\mathrm{Z}_{\mathrm{g}}$ is predicted.

The dynamical models have also been used to predict the distribution of stellar metallicities and the relation between velocity dispersion and metallicity in a region comparable with the solar neighborhood (Tinsley and Larson 1978). The slow decline in the veloctty dispersion of the gas over the lifetime of the disc leads to a relation between velocity dispersion and metallicity that is in good agreement with the data of Janes (1975), if a plausible dispersion in metallicity at a given time is assumed. Also, because of the rapid initial rise in metallicity followed by a relatively slow continuing increase, the models predict relatively few metal-poor disc stars, in agreement with observations.

\section{THE TIMESCALE FOR DISC FORMATION}

An important feature of those dynamical models that yield the best agreement with observations is the relatively long timescales involved: halo formation takes a few times $10^{9} \mathrm{yr}$, and disc formation takes an even longer time which approaches $\approx 10^{10} \mathrm{yr}$ in the outer parts because of the long time required for the gas to settle into a layer that is 
thin and dense enough for significant star formation to occur. For example, in Model 9 of Larson (1976a), the star formation rate at $15 \mathrm{kpc}$ from the center does not reach a peak until nearly $10^{10}$ yr after the beginning of the collapse. Although the exact timescales depend on model parameters, these results illustrate the possibility that many of the properties of the disc of our galaxy could result from slow disc formation by gradual infall and settling of gas into the galactic plane over a period of many times $10^{9}$ years. If this is the case, most of the stars in the outer part of the galactic disc could be considerably younger than the halo.

To distinguish between the different possibilities that have been discussed, and to reconstruct the past history of our galaxy, it is clearly necessary to have accurate information about the ages of stars and star clusters. With such information it may eventually be possible to establish the timescales for halo and disc formation and to decide to what extent various observed correlations result from secular evolution and to what extent they result from spatial variations present at a given time.

At present it is difficult to determine accurate and reliable ages for individual stars, and the most reliable age determinations are those made for clusters. New age determinations for many clusters have been made by Demarque and McClure (1977) and by Mcclure (this colloquium). They find that the ages of the metal-poor globular clusters M92 and M15 are about $15 \times 10^{9} \mathrm{yr}$, the ages of the intermediate metallicity globulars M13 and M3 are about $14 \times 10^{9} \mathrm{yr}$, and the age of the meta1rich "nuclear" globular cluster 47 Tuc is about $12 \times 10^{9} \mathrm{yr}$, with uncertainties of around $10^{9} \mathrm{yr}$. These results suggest a timescale of $\sim 3 \times 10^{9} \mathrm{yr}$ for the collapse and enrichment of the galactic halo. A more surprising result is the age of the oldest known disc cluster NGC 188, which apparently provides an upper limit for the ages of most (solar nelghborhood) disc stars; this age is found to be only $(5 \pm 1)$ $x 10^{9} \mathrm{yr}$. If there are any disc stars older than this, they must be significantly more metal-poor than NGC 188, but there are not many such metal-poor stars in solar-nelghborhood samples. For clusters younger than NGC 188, there does not appear to have been much (if any) increase of mean metallicity with time; instead, it appears that at least part of the variation in metallicity is due to spatial gradients (McClure, this colloquium).

These results suggest that the outer disc of our galaxy may be considerably younger than the halo, at least in the sense that most of the star formation did not occur until long after the formation of the halo stars. Posstbly the outer disc has formed from gas that fell in relatively recently from great distances, or possibly inefficient dissipation has caused the gas in the outer disc to take a long time to settle into a layer that is sufficiently thin and dense for star formation to become Important. It may be relevant to note that the galactic gas layer does in fact become thicker at larger radif, and that many 
spiral galaxies have gas layers that extend beyond thetr optical images and become broad and warped in the outer regions (Sancts1 1976). Whether the warps are due to the recent addition of gas or to some other disturbing influence, the gas in these regions has evidently not yet been able to settle into a thin, dense, coplanar layer, as is probably required for star formation to occur. Thus the outer parts of many spiral discs may indeed be relatively young in terms of their star formation history and stellar content, a possibility that is consistent with the fact that the colors of spiral galaxies generally become bluer with increasing radius.

\section{REFERENCES}

Audouze, J., and Tinsley, B. M., 1976. Ann. Rev. Astron. Astrophys. $14,43$.

Demarque, P., and McClure, R. D., 1977. The Evolution of Galaxies and Stellar Populations, ed. B. M. Tinsley and R. B. Larson, p. 199. Yale University Observatory, New Haven.

Eggen, 0.J., Lynden-Bel1, D., and Sandage, A. R., 1962; Astrophys. J. 136,748 .

Gott, J. R., 1977. Ann. Rev. Astron. Astrophys. 15, in press.

Janes, K. A., 1975. Astrophys. J. Suppl. 29, 161.

Larson, R. B., 1972. Nature 235, 21.

Larson, R. B., 1974. Mon. Not. Roy. Astr. Soc. 166, 585.

Larson, R. B., 1975. Mon. Not. Roy. Astr. Soc. $173,671$.

Larson, R. B., 1976a. Mon. Not. Roy. Astr. Soc. 176, 31.

Larson, R. B., 1976b. Galaxies, Sixth Advanced Course of the Swiss Society of Astronomy and Astrophysics, ed. L. Martinet and M. Mayor, p. 67. Geneva Observatory.

Larson, R. B., 1978a. J. Computational Physics, in press.

Larson, R. B., 1978b. In preparation.

Oort, J. H., 1970. Astron. Astrophys. I, 381.

Ostriker, J. P., 1977. The Evolution of Galaxies and Stellar Populations, ed. B. M. Tinsley and R. B. Larson, p. 369. Yale Untversity Observatory, New Haven.

Ostriker, J. P., and Thuan, T. X., 1975. Astrophys. J. 202, 353.

Rees, M. J., 1977. The Evolution of Galaxies and Stellar Populations, ed. B. M. Tinsley and R. B. Larson, p. 339. Yale University Observatory, New Haven.

Sancis1, R., 1976. Astron. Astrophys. 53, 159.

Searle, L., 1977. The Evolution of Galaxies and Stellar Populations, ed. B. M. Tinsley and R. B. Larson, p. 219. Yale University Observatory, New Haven.

Tinsley, B. M., and Larson, R. B., 1978. Astrophys. J., submitted. Toomre, A., 1977. The Evolution of Galaxies and Stellar Populations, ed. B. M. Tinsley and R. B. Larson, p. 401. Yale University Observatory, New Haven. 